\title{
Evaluation of photoreceptor outer segment length in hydroxychloroquine users
}

\author{
Akin Cakir $\mathbb{D}^{1} \cdot$ Şeyma Gulcenur Ozturan $\mathbb{1}^{1}$ - Dilan Yildiz ${ }^{1}$ Burak Erden ${ }^{1} \cdot$ Selim Bolukbasi ${ }^{1} \cdot$ Ethem Koray Tascilar $^{2}$. \\ Müyesser Nergiz Yanmaz ${ }^{2} \cdot$ Mustafa Nuri Elcioglu ${ }^{1}$
}

Received: 8 December 2018 / Revised: 5 March 2019 / Accepted: 10 March 2019 / Published online: 1 April 2019

(c) The Royal College of Ophthalmologists 2019

\begin{abstract}
Objective This study aims to evaluate the photoreceptor outer segment (PROS) length in patients who use hydroxychloroquine (HCQ) prior to the development of retinopathy.

Methods In this prospective, single-centre, comparative study, 44 patients using HCQ for $\geq 5$ years, 30 patients using HCQ $<5$ years, and 45 age- and sex-matched healthy controls were enrolled. The participants underwent a detailed ophthalmologic examination, spectral-domain optical coherence tomography (SD-OCT) imaging, and 10-2 automated visual field testing. The PROS length was defined as the distance between the inner surface of the ellipsoid zone and the inner surface of the retina pigment epithelium. The measurements were performed subfoveally and at 500-1000-1500 $\mu \mathrm{m}$ temporally and nasally to the foveola.

Results The mean PROS length of long-term users ( $\geq 5$ years) was statistically greater than the controls at all measurement points ( $p<0.001$ at all points). Although the subfoveal PROS length was comparable between the long-term and short-term users $(p=0.148$ ), the parafoveal PROS length measurements (nasal $1500 \mu \mathrm{m}$, nasal $1000 \mu \mathrm{m}$, nasal $500 \mu \mathrm{m}$, temporal 1000 $\mu \mathrm{m}$, and temporal $1500 \mu \mathrm{m})$ of the long-term users were significantly greater than those of the short-term users $(p<0.001$, $p=0.002, p=0.027, p=0.018, p=0.001$, respectively). No significant difference was found between the short-term users and the controls $(p=0.815, p=0.395, p=0.093, p=0.079, p=0.133, p=0.686, p=0.341$, respectively).

Conclusion The PROS length was greater in patients who used HCQ $\geq 5$ years. Possible retinal pigment epithelium toxicity may have caused this finding.
\end{abstract}

\section{Introduction}

Hydroxychloroquine sulphate (HCQ) is a synthetic derivative of quinolyl with anti-inflammatory properties that is primarily used in the treatment of rheumatic diseases, such as rheumatoid arthritis and lupus erythaematosus. Although it is a well-tolerated drug with fewer systemic toxic effects than chloroquine, retinal toxicity remains a problem in clinical practice. Recently, the American Academy of

Akin Cakir

dracakir@gmail.com

1 Department of Ophthalmology, University of Health Sciences, Okmeydani Training and Research Hospital, Istanbul, Turkey

2 Department of Internal Medicine, Division of Rheumatology, University of Health Sciences, Okmeydani Training and Research Hospital, Istanbul, Turkey
Ophthalmology (AAO) revised its recommendations on screening for chloroquine and HCQ retinopathy [1]. A high dose $(>5.0 \mathrm{mg} / \mathrm{kg}$ real weight) and a long duration of use were established as major risk factors. Additionally, concomitant renal or hepatic disease, elderly age, pre-existing maculopathy, and tamoxifen medication usage were documented as other risk factors [1-3]. Fortunately, the estimated risk of retinopathy for up to 5 years of HCQ usage has been found to be under $1 \%$ [4].

The exact mechanism of this toxicity still is unclear. Once HCQ retinopathy has occurred, it is not reversible, and the retinopathy continues to progress even in less severe cases [5]. However, when the retinopathy is recognised early, before retinal pigment epithelium (RPE) damage, a limited progression occurs after drug cessation, and the fovea can be preserved [6]. Therefore, detection in the earlier signs of retinopathy, including any alterations in the retinal layers, is essential. 
Spectral-domain optical coherence tomography (SDOCT) and visual field testing (10-2 for non-Asian patients and 24-2 for Asian patients) are the primary recommended screening tests for HCQ retinopathy [1]. Other objective tests, such as the multifocal electroretinogram and fundus autofluorescence, can also be used when needed. The predominant lesion of retinopathy in SD-OCT is a distinct focal interruption of the photoreceptor outer segment's (PROS) structural lines (disruption of the ellipsoid zone [EZ]), especially localised in the parafoveal region $[1,7]$. Thinning of the outer nuclear layer and decreased retinal thickness were demonstrated in eyes with obvious retinopathy; $[5,8]$ however, there is no consensus on the involvement of the outer and inner retina prior to the development of retinopathy. Recently, de Sisternes et al. did not find any clinically relevant changes in either the inner or outer retinal thicknesses of short-term or long-term HCQ users [9]. Conversely, Uslu et al. found thinning of the foveal PROS, a loss of the macular ganglion cell-inner plexiform layer, and RPE-Bruch's membrane thickening in patients using HCQ therapy [10].

Considering that the photoreceptors are the primary targeted cells of toxicity, the evaluation of the PROS length in the parafoveal region may provide earlier detection of the beginning stages of retinopathy. Thus, in the current study, we aimed to investigate whether the PROS length differed among HCQ users according to HCQ dosage and duration of use as well as to compare those users with healthy subjects.

\section{Materials and methods}

The study was conducted in the Ophthalmology Department of the University of Health Sciences, Okmeydani Training and Research Hospital, Istanbul, Turkey. It was approved by the Clinical Research Ethics Committee of the Marmara University Faculty of Medicine and adhered to the principles of the Declaration of Helsinki. All participants signed a written informed consent form before enrolment. All of the patients had a diagnosis of rheumatoid arthritis (RA) and/or lupus erythaematosus, based on the 2010 American College of Rheumatology (ACR)/European League Against Rheumatism (EULAR) classification criteria for RA, the 1987 ACR criteria, the 2012 Systemic Lupus International Collaborating Clinics' criteria, and the 1997 ACR criteria.

Prior to the study, we computed the required sample size by using the G*Power 3.1.9.2 (Universitat Düsseldorf) programme. We calculated that the study had to recruit a minimum of 31 individuals for each group to have $95 \%$ power with a $5 \%$ type 1 error level. We achieved the effect size via an automatic programme embedded within $\mathrm{G}^{*}$ power by providing the estimated mean and standard deviation values (we used $\sim 46-49 \mu \mathrm{m}$ for the mean of the PROS length and $3 \mu \mathrm{m}$ for the standard deviation and found an effect size of 0.95).

Patients who received consultation for HCQ retinopathy at our clinic and met the inclusion criteria were included in this prospective comparative study. According to the duration of HCQ use, the 74 HCQ users were divided into two groups. Those who had used HCQ for less than five years $(n=30)$ constituted the short-term user group, and those who had used HCQ for more than 5 years $(n=44)$ constituted the long-term user group. Forty-five age- and sex-matched healthy subjects were enrolled as a control group. The exclusion criteria included any refractive errors (spherical or cylindrical) greater than \pm 3.0 diopters, the presence of any pathology of the anterior segment or fundus (e.g. pre-existing maculopathy), a history of ocular trauma or ocular surgery, and a history of chronic ocular diseases, such as glaucoma, keratoconus, and uveitis.

All participants underwent a comprehensive ophthalmologic examination, including best corrected visual acuity (BCVA) testing using the logarithm of the minimum angle of resolution (logMAR), an anterior segment biomicroscopy, an intraocular pressure measurement, a dilated fundoscopy, spectral-domain optical coherence tomography (SD-OCT) imaging, and 10-2 automated visual field testing. Participants were asked about their daily dose of HCQ, duration of HCQ usage, concomitant hepatic or kidney disease, and tamoxifen or other diseasemodifying drug usage. The HCQ cumulative dose $(\mathrm{g})$ was calculated by multiplying the current daily dose (g/day) by the duration of HCQ use (years). Perimetry was performed using a standard 10-2 Humphrey Visual Field Analyzer (Humphrey Instruments, Inc., San Leandro, CA) with a white test spot. Patients with any distinct pathologies in the visual field, indicating HCQ retinopathy, were excluded from the study.

SD-OCT images were obtained through Spectralis OCT (Spectralis; Heidelberg Engineering, Heidelberg, Germany) by a certified operator. The following scan acquisition parameters were required: a dense volume scan $\left(30 \times 25^{\circ}\right.$, $\sim 9 \times 7.5 \mathrm{~mm}$ ); $31 \mathrm{~B}$-scans, each spaced $244 \mathrm{\mu m}$ apart; an automatic real-time mean of 20; and a high speed (512 Ascans/B-scan). Automatic segmentation of all retinal layers was achieved by the embedded programme in Spectralis. The PROS length was defined as the distance from the inner surface of the ellipsoid zone to the inner surface of the RPE. The Early Treatment Diabetic Retinopathy Study (ETDRS) inlay with a central circle ( $1 \mathrm{~mm}$ or $500 \mu \mathrm{m}$ in diameter) that was centred on the foveola, a concentric inner ring $(2 \mathrm{~mm}$ or $1000 \mu \mathrm{m}$ in diameter), and a concentric outer ring ( $3 \mathrm{~mm}$ or $1500 \mu \mathrm{m}$ in diameter) was used to achieve standardised measurement points (Fig. 1). The measurements were performed subfoveally as well as at 500,1000 , and $1500 \mu \mathrm{m}$ 


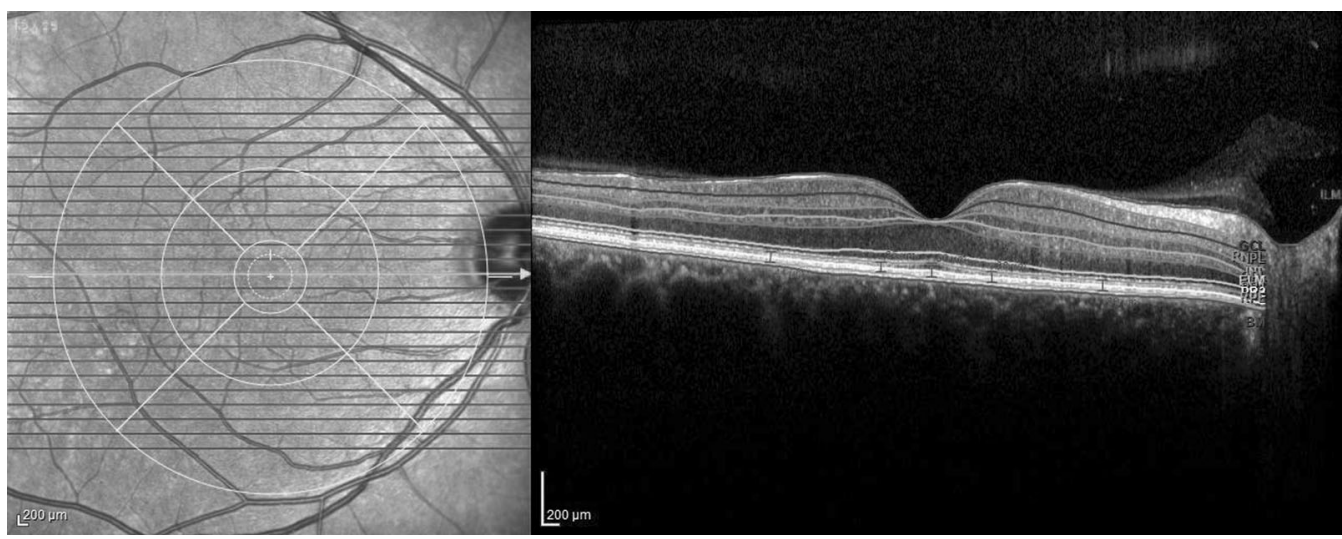

Fig. 1 Reference sample figure for measurement of photoreceptor outer segments at different locations by using the Early Treatment Diabetic Retinopathy Study (ETDRS) inlay

nasally and temporally to the fovea by two independent and experienced observers who were masked to the patients' information. Each observer performed the measurements in two different sessions. The average values (first, the average of the two sessions of each observer and, then, the average of the two observers) were used for statistical analyses. Although both eyes were measured, only the right eyes of subjects were studied. Since there were two observers, the inter- and intra-observer variability was investigated. Additionally, an inter-sessional variability for each observer was also calculated.

Inter-observer reliability was calculated by comparing the two observers' measurements in all measurement areas (nasal 1500, nasal 1000, nasal 500, subfoveal, temporal 500 , temporal 1000, and temporal 1500) by Student's $t$-test, which yielded $p=0.164, p=0.503, p=0.94, p=0.113$, $p=0.438, p=0.96$, and $p=0.64$, respectively. Intraobserver variability was assessed by the coefficient of variance $(\% \mathrm{CV})$ at all measurement points. The $\mathrm{CV}$ was calculated by using the following formula: $\mathrm{CV}=\mathrm{SD} /$ mean $\times 100$. The first observer's CVs were $5.8 \%, 7.9 \%$, $6.2 \%, 7.1 \%, 6.4 \%, 6.5 \%$, and $6.3 \%$, respectively, and the second observer's CVs were 6.3\%, 8.4\%, 7.3\%, 6.3\%, $7.1 \%, 6.6 \%$, and $7.3 \%$. Inter-sessional variability was analysed by using the Wilcoxon signed ranks test. The variation between the sessions of each observer was not statistically significant $(p>0.05$ for all measurement areas).

Statistical analyses were performed using the IBM SPSS software version 25.0 (SPSS Inc., Chicago, IL, USA). Since the PROS length measurements were not normally distributed, the Mann-Whitney U test was used to compare the parameters. Predictors with a $p$ value of $<0.05$ in the univariate analysis were included in the final multiple linear regression model. A forward stepwise procedure was used. A $p$ value of $<0.05$ was used to infer statistical significance.

\section{Results}

A total of 119 eyes of the 119 participants were studied. Forty-four subjects had used HCQ for more than five years (long-term users), 30 subjects had used HCQ for under five years (short-term users), and 45 healthy subjects were used as controls. The groups were similar in terms of age, sex, spherical equivalence, and axial length $(p=0.298, p=$ $0.197, p=0.075$, and $p=0.808$, respectively). There were only one $(2.2 \%)$ male patient in the long-term user group and two $(6.6 \%)$ male patients in the short-term user group. Among the long-term users, six $(13.6 \%)$ patients had concomitant kidney disease, and four $(9 \%)$ patients had hepatic disease. Two $(6.6 \%)$ and three (10\%) patients, respectively, had these conditions in the short-term user group. No participant had a history of tamoxifen use. The baseline clinical features of the subjects are summarised in Table 1 .

The mean PROS length of the long-term users was significantly greater than the controls at all measurement points ( $p<0.001$ in all quadrants; Fig. 2; Table 2). There were statistically significant differences in the PROS lengths measured at nasal 1500, nasal 1000, nasal 500, temporal 1000, and temporal 1500 between the long-term and shortterm users. The parafoveal PROS length measurements of the long-term users were significantly greater than the shortterm users $(p<0.001, p=0.002, p=0.027, p=0.018$, and $p=0.001$, respectively; Fig. 2; Table 2). However, the subfoveal PROS length did not differ between the long-term and short-term users $(p=0.148)$. Additionally, there was no significant difference between the controls and the shortterm users in terms of the PROS length at any measurement point $(p=0.815, p=0.395, p=0.093, p=0.079, p=$ $0.133, p=0.686$, and $p=0.341$, respectively; Fig. 2; Table 2).

The duration and dose had a significant positive correlation with the PROS length in univariate analysis. A multiple regression model identified duration as a predictor 
Table 1 Baseline clinical features of the subjects

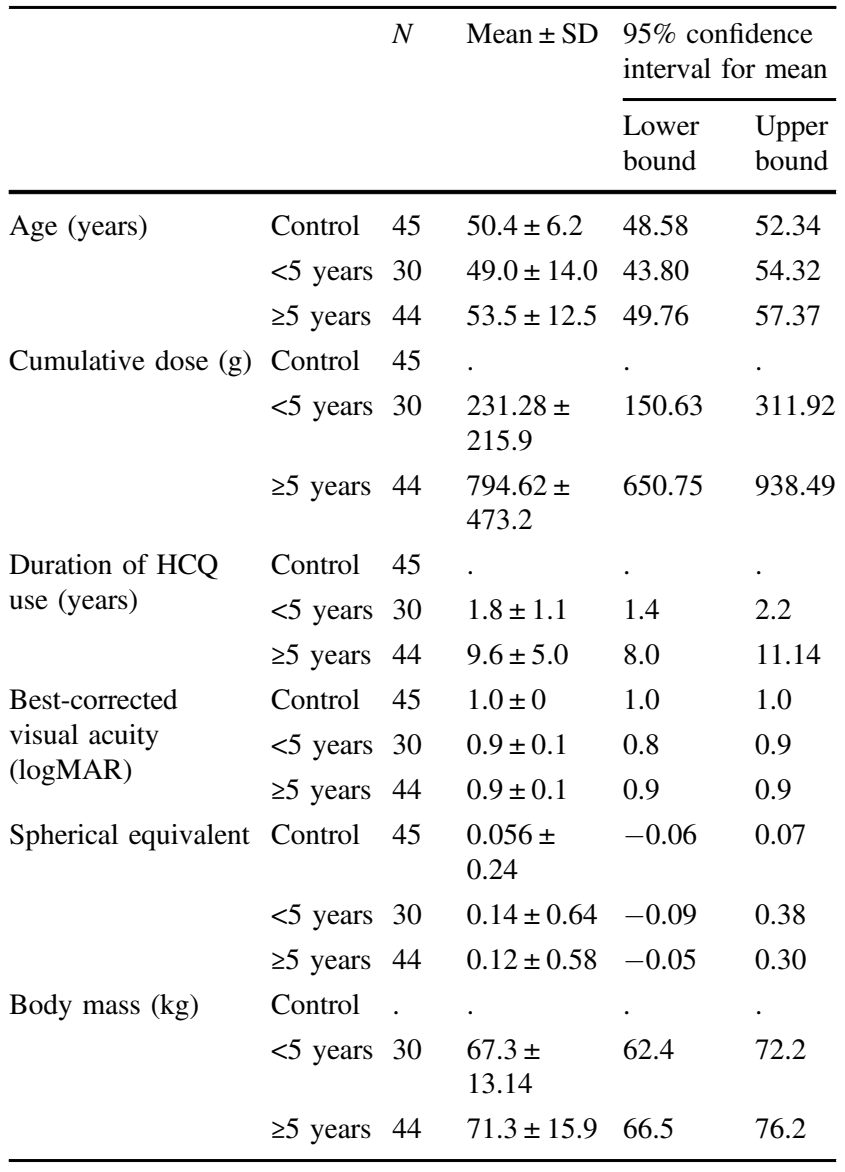

of the PROS length at all measurement points except for the subfoveal area (B coefficient: 0.259, $p<0.001$; B coefficient: 0.182, $p=0.008$; B coefficient: $0.207, p=0.003$; B coefficient: $0.191, p=0.001$; B coefficient: $0.211, p<$ 0.001 ; and B coefficient: $0.320, p<0.001$, respectively). Furthermore, the HCQ cumulative dose was found to be a predictor for the subfoveal PROS length (B coefficient: $2.145, p<0.010)$.

\section{Discussion}

This study showed an elongation of the PROS length in long-term HCQ users prior to the development of retinopathy. This may be a precursor finding of photoreceptor damage at an early stage of HCQ retinopathy. The exact target tissue of HCQ in the human retina is still a matter of debate. In animal models, chloroquine accumulates in the RPE and leads to a disruption of the lysosomal function [11, 12]. RPE cells are responsible for phagocytosis of the PROS; to maintain vision, outer segments must be renewed periodically, and thereby, the proper length of the PROS is maintained by that coordinated phagocytosis activity. Thus,

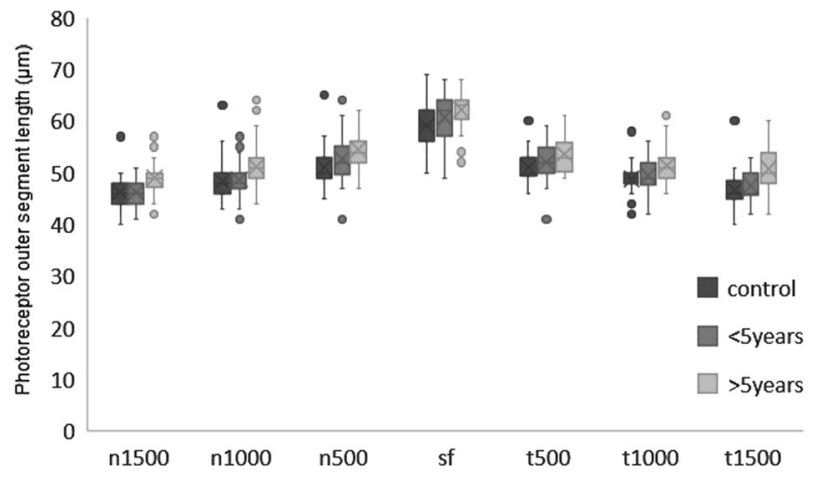

Fig. 2 Box plot graphic showing the mean photoreceptor outer segment lengths of all the subjects at entire measurement points $(\mathrm{n} 1500=$ $1500 \mu \mathrm{m}$ nasally to fovea, $\mathrm{n} 1000=1000 \mu \mathrm{m}$ nasally to fovea, $\mathrm{n} 500=$ $500 \mu \mathrm{m}$ nasally to fovea, sf subfoveal, $\mathrm{t} 15001500 \mu \mathrm{m}$ temporally to fovea, t1000 $1000 \mu$ temporally to fovea, t500 $500 \mu \mathrm{m}$ temporally to fovea)

any damage in the lysosomal and/or enzymatic activity of the RPE may initially result in the elongation of the PROS, subsequent shortening and, eventually, the loss of photoreceptors.

A different perspective on this elongation is as follows. A compensatory elongation may result from early and subtle loss of neighbouring photoreceptors. As Stepien et al. showed, there is some dropout in cone count as well as a simultaneous increase in cone spacing through the use of adaptive optics in HCQ retinopathy at a preclinical stage [13]. Similar results were confirmed by a following study [14].

Several other explanations of the elongation of the PROS are possible, including outer segment membrane stabilisation and reduced RPE phagocytosis, which are independent of RPE lysosomal stabilisation/enzyme disruption. We found that long-term HCQ users had a greater PROS length than short-term users in the parafoveal region but not in the fovea. This is compatible with the current data in the literature. We attribute this finding to the fact that the retinopathy tended to arise in the parafoveal distribution, as expected.

Several studies have concentrated on the retinal thickness and HCQ usage [5, 8, 10, 15-17]. The majority did not focus on the PROS specifically, and many reported conflicting results. While some publications have reported a progressive thinning of either the inner or outer retinal thickness, others have not shown any changes. Increased retinal thickness in the parafoveal areas in the short-term (6 months after starting HCQ treatment) was observed in only one study [17]. It is likely that the first reason for the difference between those studies is the existence of an already established retinopathy. There is almost a consensus on retinal thinning in the eyes with obvious retinopathy. Therefore, we intentionally excluded the patients who had clinically documented retinal toxicity. The second reason 
Table 2 The mean photoreceptor outer segment length (PROS) of the subjects

\begin{tabular}{|c|c|c|c|c|c|}
\hline \multicolumn{2}{|c|}{$\overline{\text { PROS length }(\mu \mathrm{m})}$} & \multirow[t]{2}{*}{$\mathrm{N}$} & \multirow[t]{2}{*}{ Mean } & \multicolumn{2}{|c|}{$95 \%$ confidence interval for mean } \\
\hline & & & & Lower bound & Upper bound \\
\hline \multirow[t]{3}{*}{ Nasal1500 } & Control & 45 & $46.13 \pm 3.0$ & 45.22 & 47.04 \\
\hline & $<5$ years & 30 & $46.16 \pm 2.8$ & 45.11 & 47.22 \\
\hline & $\geq 5$ years & 44 & $49.05 \pm 3.1$ & 48.09 & 50.01 \\
\hline \multirow[t]{3}{*}{ Nasal1000 } & Control & 45 & $48.24 \pm 3.5$ & 47.17 & 49.31 \\
\hline & $<5$ years & 30 & $48.73 \pm 3.8$ & 47.29 & 50.17 \\
\hline & $\geq 5$ years & 44 & $51.20 \pm 4.1$ & 49.94 & 52.46 \\
\hline \multirow[t]{3}{*}{ Nasal500 } & Control & 45 & $51.06 \pm 3.6$ & 49.98 & 52.15 \\
\hline & $<5$ years & 30 & $52.60 \pm 4.7$ & 50.83 & 54.36 \\
\hline & $\geq 5$ years & 44 & $54.50 \pm 3.5$ & 53.40 & 55.59 \\
\hline \multirow[t]{3}{*}{ Subfoveal } & Control & 45 & $59.15 \pm 4.1$ & 57.91 & 60.39 \\
\hline & $<5$ years & 30 & $60.73 \pm 4.7$ & 58.95 & 62.51 \\
\hline & $\geq 5$ years & 44 & $62.17 \pm 3.5$ & 61.09 & 63.24 \\
\hline \multirow[t]{3}{*}{ Temporal500 } & Control & 45 & $51.04 \pm 2.8$ & 50.17 & 51.91 \\
\hline & $<5$ years & 30 & $51.96 \pm 3.6$ & 50.59 & 53.34 \\
\hline & $\geq 5$ years & 44 & $53.60 \pm 3.3$ & 52.57 & 54.62 \\
\hline \multirow[t]{3}{*}{ Temporal1000 } & Control & 45 & $48.84 \pm 2.9$ & 47.96 & 49.72 \\
\hline & $<5$ years & 30 & $49.40 \pm 3.0$ & 48.27 & 50.52 \\
\hline & $\geq 5$ years & 44 & $51.40 \pm 3.4$ & 50.34 & 52.46 \\
\hline \multirow[t]{3}{*}{ Temporal1500 } & Control & 45 & $46.64 \pm 3.3$ & 45.62 & 47.65 \\
\hline & $<5$ years & 30 & $47.50 \pm 3.0$ & 46.34 & 48.65 \\
\hline & $\geq 5$ years & 44 & $50.72 \pm 4.1$ & 49.47 & 51.98 \\
\hline
\end{tabular}

for this difference may have arisen from evaluating the average retinal thickness. It must be considered that evaluating the retinal thickness in a layer-by-layer manner will lead to more accurate conclusions because a minimal change in one layer may not be discovered when summing up the layers into overall thickness.

Modi et al. [16], for instance, demonstrated that HCQ toxicity results in both outer and inner retinal volumetric thinning compared to age-matched control patients and patients taking HCQ but not manifesting toxicity. It must be emphasised that in their study, the authors did not measure the PROS length but rather the outer retina (outer plexiform layer-RPE), and this thinning was found only in the patients with obvious HCQ toxicity. Moreover, Pasadhika et al. [15] showed a thinning of the perifoveal inner retinal layers even in the absence of functional or structural clinical changes involving the photoreceptor or RPE cell layers. The authors did not find any comparable change in the photoreceptor outer segment (POS) thickness [15]. This may have resulted from the different measurement methods that they used (e.g. a specific software programme) because they reported the average mean POS thickness as $\sim 35 \mu \mathrm{m}$. In comparison, in our study, it was $\sim 45 \mu \mathrm{m}$.

Our findings also revealed that the duration of use and the cumulative dose were significant predictors of the PROS length. These have been major risk factors for toxicity in previous reports [1-3]. However, our study was the first to evaluate the parafoveal PROS and to document an increase in the PROS length. Additionally, we studied only one eye of each patient to eliminate the magnification of statistical significance.

The main limitation of this report is the absence of an automatic measurement programme embedded in the SDOCT, as in the retinal nerve fibre layer, ganglion cell layer, or internal plexiform layer analysis. Nevertheless, the superior and inferior borders (EZ and RPE) were segmented automatically, and inter- and intra-observer variability was within normal ranges. Some studies have also claimed that manual measurements are still superior to automated measurements [18, 19]. The actual disease (rheumatoid arthritis or systemic lupus erythaematosus) may have contributed to our results in a limited way, since retinal changes are very rare in rheumatologic diseases [20]. For this reason, we believe that our results are reliable.

In conclusion, the PROS length increases in long-term HCQ users over time even in the absence of an obvious retinopathy. This finding may play a complementary role in the further investigation of the patients. Well-designed prospective cohort studies are recommended to validate these results. 


\section{Summary}

\section{What was known before}

- The status of photoreceptor prior to development of hydroxychloroquine retinopathy was unclear.

\section{What this study adds}

- This study showed an elongation of PROS length in the long-term HCQ users prior to development of retinopathy

\section{Compliance with ethical standards}

Conflict of interest The authors declare that they have no conflict of interest.

Publisher's note: Springer Nature remains neutral with regard to jurisdictional claims in published maps and institutional affiliations.

\section{References}

1. Marmor MF, Kellner U, Lai TY, Melles RB, Mieler WF, American Academy of Ophthalmology. Recommendations on screening for chloroquine and hydroxychloroquine retinopathy [2016 Revision]. Ophthalmology. 2016;123:1386-94.

2. Melles RB, Marmor MF. The risk of toxic retinopathy in patients on long-term hydroxychloroquine therapy. JAMA Ophthalmol. 2014;132:1453-60.

3. Marmor MF, Kellner U, Lai TY, Lyons JS, Mieler WF, American Academy of Ophthalmology. Revised recommendations on screening for chloroquine and hydroxychloroquine retinopathy. Ophthalmology. 2011;118:415-22.

4. Wolfe F, Marmor MF. Rates and predictors of hydroxychloroquine retinal toxicity in patients with rheumatoid arthritis and systemic lupus erythematosus. Arthritis Care Res [Hoboken]. 2010;62:775-84.

5. Lally DR, Heier JS, Baumal C, Witkin AJ, Maler S, Shah CP, et al. Expanded spectral domain-OCT findings in the early detection of hydroxychloroquine retinopathy and changes following drug cessation. Int J Retina Vitreous. 2016;18:18.

6. Marmor MF, Hu J. Effect of disease stage on progression of hydroxychloroquine retinopathy. JAMA Ophthalmol. 2014;132: 1105-12.
7. Marmor MF. Comparison of screening procedures in hydroxychloroquine toxicity. Arch Ophthalmol. 2012;130:461-9.

8. Cukras C, Huynh N, Vitale S, Wong WT, Ferris FL 3rd, Sieving PA. Subjective and objective screening tests for hydroxychloroquine toxicity. Ophthalmology. 2015;122:356-66.

9. de Sisternes L, Hu J, Rubin DL, Marmor MF. Analysis of inner and outer retinal thickness in patients using hydroxychloroquine prior to development of retinopathy. JAMA Ophthalmol. 2016. https://doi.org/10.1001/jamaophthalmol.2016.0155

10. Uslu H, Gurler B, Yildirm A, Tatar MG, Aylin Kantarci F, Goker $\mathrm{H}$, et al. Effect of hydroxychloroquine on the retinal layers: a quantitative evaluation with spectral-domain optical coherence tomography. J Ophthalmol. 2016. https://doi.org/10.1155/2016/ 8643174.

11. Rosenthal A, Kolb H, Bergsma D, Huxsoll D, Hopkins JL. Chloroquine retinopathy in the rhesus monkey. Invest Ophthalmol Vis Sci. 1978;17:1158-75.

12. Mahon GJ, Anderson HR, Gardiner TA, McFarlane S, Archer DB, Stitt AW. Chloroquine causes lysosomal dysfunction in neural retina and RPE: implications for retinopathy. Curr Eye Res. 2004;28:277-84.

13. Stepien KE, Han DP, Schell J, Godara P, Rha J, Carroll J. Spectraldomain optical coherence tomography and adaptive optics may detect hydroxychloroquine retinal toxicity before symptomatic vision loss. Trans Am Ophthalmol Soc. 2009;107:28-33.

14. Debellemaniere G, Flores M, Tumahai P, Meillat M, Bidaut Garnier M, Delbosc B, et al. Assessment of parafoveal cone density in patients taking hydroxychloroquine in the absence of clinically documented retinal toxicity. Acta Ophthalmol. 2015;93:534-40.

15. Pasadhika S, Fishman GA, Choi D, Shahidi M. Selective thinning of the perifoveal inner retina as an early sign of hydroxychloroquine retinal toxicity. Eye [Lond].. 2010;24:756-62.

16. Modi YS, Au A, Parikh VS, Ehlers JP, Schachat AP, Singh RP. Volumetric single-layer inner retinal analysis in patients with hydroxychloroquine toxicity. Retina. 2016;36:1941-50.

17. Yülek F, Uğurlu N, Akçay E, Kocamış Sİ, Gerçeker S, Erten Ş, et al. Early retinal and retinal nerve fiber layer effects of hydroxychloroquine: a follow up study by sdOCT. Cutan Ocul Toxicol. 2013;32:204-9.

18. Taban M, Sharma S, Williams DR, Waheed N, Kaiser PK. Comparing retinal thickness measurements using automated fast macular thickness map versus six-radial line scans with manual measurements. Ophthalmology. 2009;116:964-70.

19. Heussen FM, Ouyang Y, McDonnel EC, Narala R, Ruiz-Garcia $\mathrm{H}$, Walsh $\mathrm{AC}$, et al. Comparison of manually corrected retinal thickness measurements from multiple spectral-domain optical coherence tomography instruments. $\mathrm{Br} \mathrm{J}$ Ophthalmol. 2012;96:380-5.

20. Gokmen O, Yesilirmak N, Akman A, Gür Güngör S, Yücel AE, Yeşil $\mathrm{H}$, et al. Corneal, scleral, choroidal and foveal thickness in patients with Rheumatoid arthritis. Turk J Ophthalmol. 2017;47:315-9. 